\title{
FAST DETERMINATION OF ATTENUATION FROM MICROSEISMICITY FOR LARGE DATASETS
}

\author{
Milosz WCISŁO $^{1,2)} *$ and Leo EISNER ${ }^{3)}$ \\ ${ }^{1)}$ Institute of Rock Structure and Mechanics, CAS, V Holešovičkách 41, 18209 Praha 8, Czech Republic \\ ${ }^{2)}$ Charles University in Prague, Faculty of Mathematics and Physics, V Holešovičkách 2, 18000 Praha 8, Czech Republic \\ ${ }^{3)}$ Seismik LTD, Kubišova 1265/8, 18200 Praha 8, Czech Republic
}

*Corresponding author's e-mail: wcislo@irsm.cas.cz

\begin{tabular}{l} 
ARTICLE INFO \\
\hline Article history: \\
Received 20 December 2018 \\
Accepted 28 May 2019 \\
Available online 18 June 2019 \\
\hline
\end{tabular}

Keywords:

Seismic attenuation

Body waves

Induced seismicity

Fourier analysis

\begin{abstract}
We develop a robust and automatic determination of peak frequencies of direct wave arrivals from microseismic events using mirror image of the recorded signal. These peak frequencies can be then used to evaluate attenuation quality factor $(\mathrm{Q})$. We propose to use procedure that consists of automatic determination of the peak frequencies in combination with matched filter to facilitate selection of suitable events. Using both natural and induced seismicity datasets, we show that matched filter detection along with automatic determination of the peak frequencies gives consistent values of quality factor when compared with manual processing. Proposed technique significantly reduces processing time making it a suitable tool in attenuation analysis of large datasets.
\end{abstract}

\section{INTRODUCTION}

Seismic attenuation is an important characteristic of the medium. It is used in forward and inverse modelling (e.g. Guo and McMechan, 2017; Mousavi et al., 2017), determination of feasibility of detection of seismic events (e.g. Hallo, 2012; Wüstefeld et al., 2014) as well as interpretation of the medium properties itself. For example, a high attenuation is an indication of water and oil saturation (Pham et al., 2002), while presence of methane or compressed $\mathrm{CO}_{2}$ results in even stronger attenuation (Castiello et al., 2015). Because of this attenuation is attracting significant attention of the oil and gas industry. Additionally, an attenuation model is needed for source mechanism inversions (e.g. Jechumtalova and Eisner, 2008) and any amplitude based inversion (such as AVO or AVA).

Eisner et al. (2013) developed a reliable method of the effective attenuation quality factor (Q) measurement from microseismicity using the peak frequency of direct waves. The method is similar to the rise time method (e.g. Tselentis et al., 2010). It is a single station measurement, unlike to the frequently used attenuation determination from multiple receivers such as instantaneous frequency method (Matheney and Nowack, 1995), frequency shift method (Quan and Harris, 1997) or probably the most popular spectral ratio method (e.g. Tonn, 1989;
Beckwith et al., 2017). The single receiver inversion is useful for sparse networks where receivers are far apart (e.g., sparse surface network) or where they are placed in different geological conditions (e.g., downhole arrays).

Determination of seismic attenuation requires significant involvement of interpreter even if as simple method as the peak frequency is used. Manual processing in case of large datasets can require significant amount of time, especially if the big portion of arrivals are noisy and cannot be used in peak frequency analysis. This study addresses semiautomated procedure that involves evaluation of the peak frequencies from waveforms selected through matched filter in case of large datasets acquired in microseismic monitoring. We test this method on both synthetic and two real datasets and compare manual and automated determination of attenuation.

\section{METHOD}

Eisner et al. (2013) showed that the peak frequency $f_{\text {peak }}$ of the body waves radiated from a shear source in an isotropic medium can be related to the global absorption factor $t^{*}$ (Červený, 2001) by a simple equation:

$$
f_{\text {peak }}=\frac{1}{\pi t^{*}}
$$



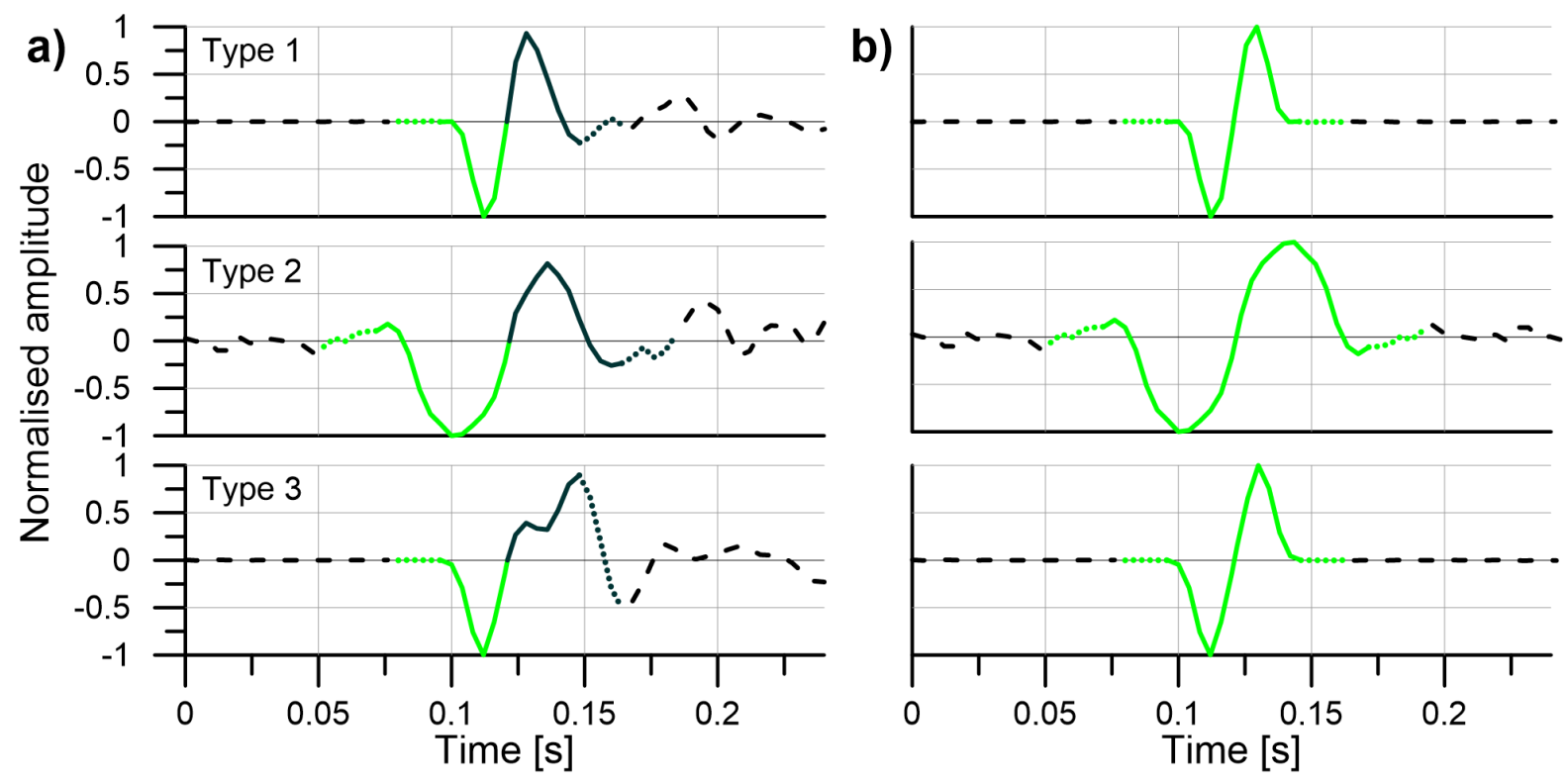

Fig. 1 a) Three examples of waveforms suitable for determination of $Q$ factor using the peak frequency. Each waveform has first half period of the arrival relatively unaffected by noise, but Type 2 and in particular Type 3 waveform after zero crossing is influenced by further arrivals. Bright and dark parts with continuous and dotted line indicate the time intervals used for the Fourier transform of each waveform. Cosine taper is applied in the time intervals with dots.

b) Waveforms obtained with mirror imaging of the first half period of signals shown in a) i.e. the second part of the signal is replaced by the mirror image of the first half-period of the signal.

The peak frequency is the frequency of the maximum amplitude of ground velocity spectra of a signal. If the attenuation quality factor in a given medium is constant, Equation (1) may be further simplified and re-arranged to obtain the effective, i.e., constant (Kjartansson, 1979), attenuation quality factor:

$Q=\pi f_{\text {peak }} T$

where $T$ is the traveltime of the wave (e.g., P- or Swave). The Equations (1) and (2) are valid for the peak frequencies below the corner frequency of a seismic event (Eisner et al., 2013), therefore their application is limited to weak microseismic events with corner frequencies significantly higher than the observed $f_{\text {peak }}$. Wcisło and Eisner (2016) showed that the peak frequency can be reliably determined from the first half-period of the signal. This method provides more stable results than the peak frequency determined from the Fourier transform of the time interval corresponding to the whole arrival. It is because we avoid later (scattered) arrivals interfering with the further part of the direct arrival. In Figure 1a we show 3 examples of direct arrivals of microseismic events. Figure 1a (top panel) shows an observed direct wave signal without the influence of the later arrivals (we shall call it the Type 1 waveform in this study). Such waveform yields virtually the same peak frequency measurement for both the first half-period and Fourier transform of the whole waveform. Other example of observed arrival - Figure 1a (middle panel) - include limited distortion - we shall call it the Type 2 waveform. At last - Figure 1a (bottom panel) shows waveform with later arrival(s) that significantly interfere with direct wave in the second half-period of the direct wave signal - we shall call it the Type 3 waveform. The influence of interference would result in significant error in the peak frequencies determined from the Fourier transform of the whole signal, nevertheless manually determined half period of the signal can be used for the peak frequency determination.

Even though the manual determination of peak frequency in the time domain is easy for individual events, it is time consuming to carry out for large dataset. The automation of the process that would include suitable event selection and determination of peak frequency is subject of this study. Initially let us assume we can select from a dataset events with waveform of Types 1-3 (we will show later one possible method of such selection - the matched filter). Let us now discuss automated peak frequency determination as it is difficult to consistently autopick the onset of the arrival. On the other hand, the whole waveforms of the Type 2 or the Type 3 are not suitable for reliable determination of the peak frequencies from the Fourier spectra of the whole direct arrival. The Fourier spectrum is affected by interfering waves - we show such a spectrum in Figure 2a (Type 3 waveform). Note that the time intervals with data were zero padded before applying fast Fourier transform resulting in smoothness of the observed spectra. The influence of multiple waves 
a)

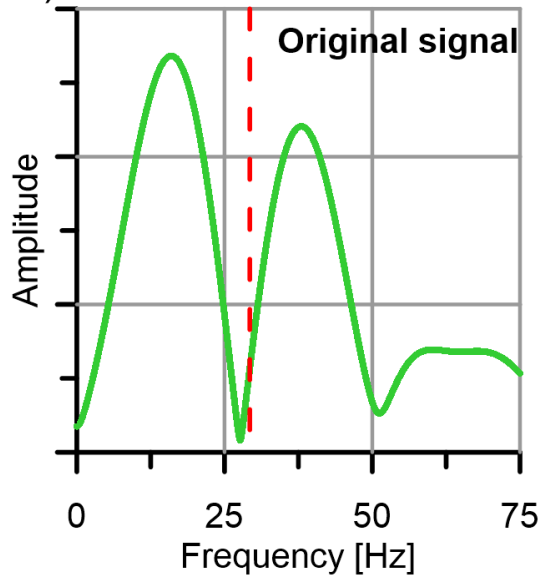

b)

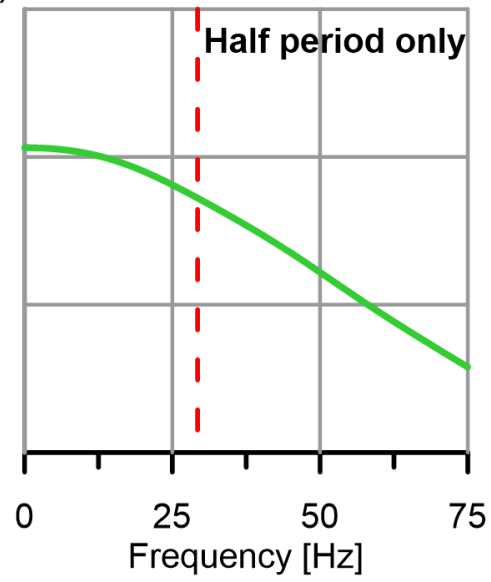

c)

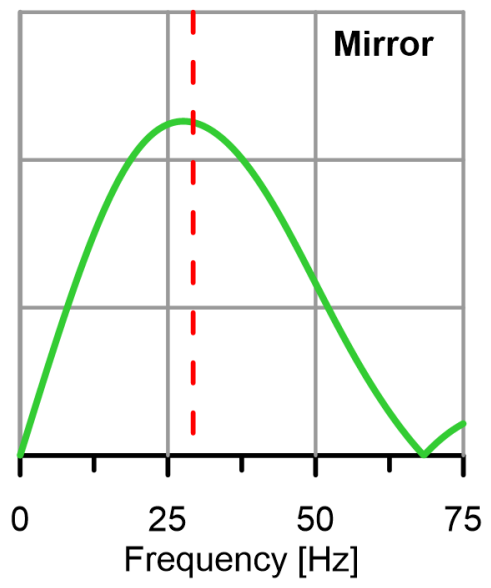

Fig. 2 Amplitude spectra of the waveform the Type 3 of Figure 1 calculated using original signal (panel a), cut half period (panel b) and mirrored signal (panel c). Dashed vertical line indicates the peak frequency calculated using manual picking of the half period.

interfering with direct arrival is a problem commonly encountered especially in downhole microseismic monitoring where guided horizontally propagating waves interfere. In case of large datasets, many waveforms are likely to have second half-period affected by the interference which would make the use of the Fourier spectrum questionable. Alternatively, one could consider applying Fourier transform only to the first part of the waveform. Unfortunately, it is not convenient approach due to the properties of Fourier transform. To understand this let's use standard definition of the Fourier spectrum $\mathrm{S}(\mathrm{f})$ of the signal $s(t)$ :

$$
S(f)=\int_{-\infty}^{\infty} s(t) \exp (-i 2 \pi t f) d t
$$

For zero frequency the Fourier transformation is equal to the integral over the signal. The integral over the first half-period of the signal has significant non zero value. In such case, the signal's integral likely dominates the amplitude spectra and the peak frequency of the amplitude spectrum is at zero or near zero frequency. Figure $2 \mathrm{~b}$ illustrates the described issue using as an example spectra of the Type 3 waveform. The peak of the spectrum of the raw signal (left panel) does not agree with the peak frequency measured manually from the first half-period due to interference with later arrival, the spectrum of the signal zeroed after the first half-period, is dominated by the DC component (mean amplitude of the signal).

To resolve this problem and to allow calculation of the peak frequency in the frequency domain we propose Fourier spectrum calculation allowing use of the Types 2 and 3 waveforms by using modified waveforms where the second half-period of the signal is replaced by a mirror image (mirrored around the zero axis) of the first half-period. In this way we create the signal that can be used in frequency domain to calculate the peak frequency corresponding to the first half-period. It eliminates the above discussed issue with the DC component as the integral over the newly created signal is negligible. Figure $1 \mathrm{~b}$ illustrates modified mirrored waveforms corresponding to those presented in Figure 1a, that can be further used to determine the peak frequency using the Fourier transformation. Fourier spectrum of created mirrored signal is shown in Figure 2c, we can see that frequency that corresponds to maximum amplitude of the spectrum is close to the peak frequency obtained with the use of half-period measured in the time domain.

Note that to create a reliable mirrored signal we need to follow a few common-sense steps. First of all, we need to consider the fact that the mirroring is made at the point of the zero crossing. Therefore, determination of this point is crucial. We need to remove the mean of the signal interval as a non-zero mean may result in distortion of the zero-crossing. This can be also ensured by appropriate filtering. We also need to consider the fact that signals we are dealing with are discrete. It means that the zero crossing will not be sampled, and that simply mirrored signal with samples taken from the original seismogram will produce signal which will not be equispaced in the vicinity of the zero-crossing. One can work with non-equispaced versions of the FFT algorithm, although, it is much easier to interpolate the whole mirrored signal interval and apply the usual FFT algorithms. In our study we use cubic spline interpolation that is in common use in signal processing due to continuous second derivatives of the interpolated signal that it provides. If our signal is relatively poorly sampled and the first half-period is registered by e.g. only 3 samples it may be good to upsample the signal in frequency domain first, and only then proceed with the mirroring of the signal and applying interpolation. This approach reduces possible bias that may be introduced by numerical 
a)
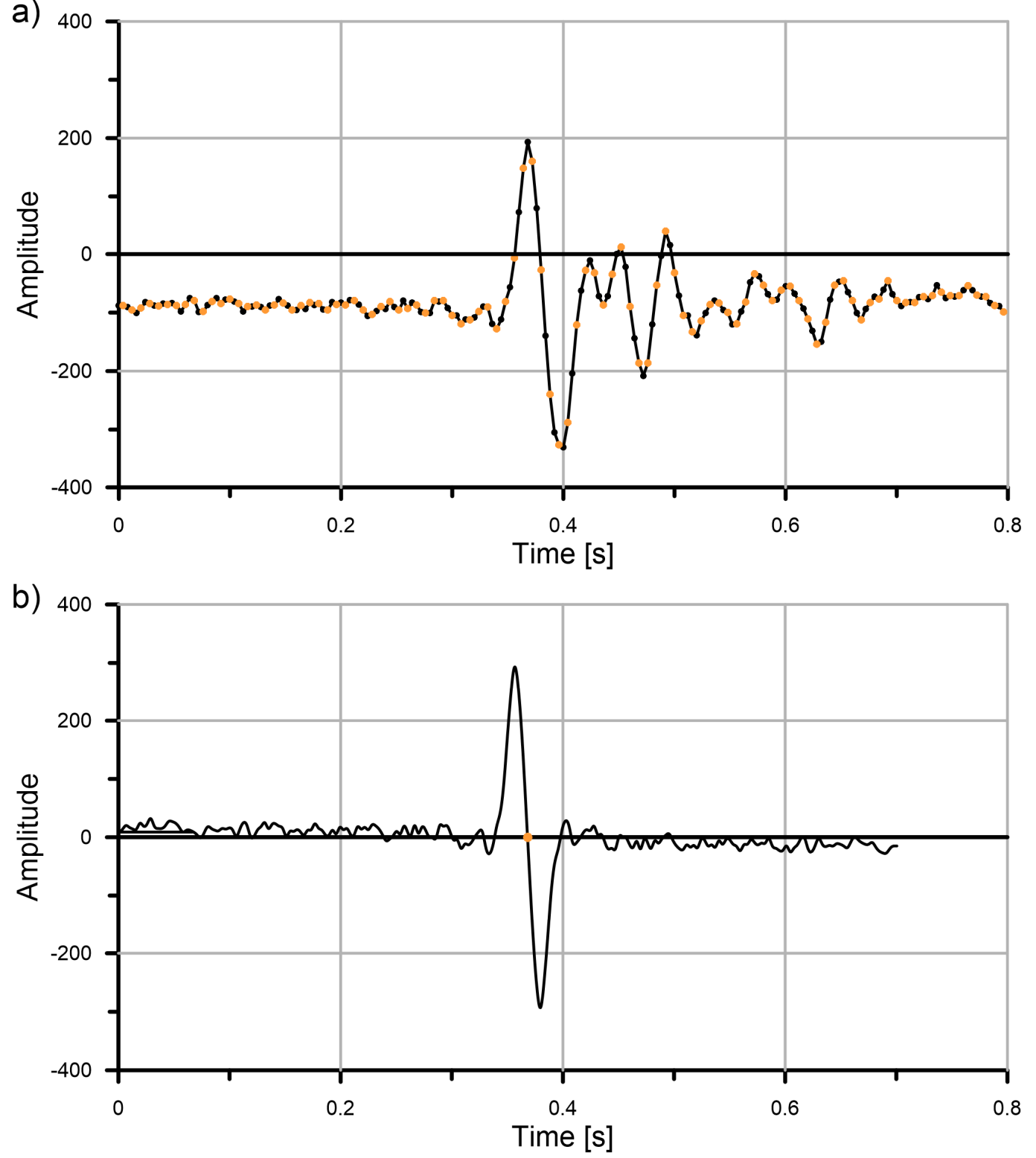

Fig. 3 Example of preparation of waveform allowing the use of mirror technique; a) original signal (bright dots) along with result of upsampling in frequency domain (black dots); b) signal after removal of the mean, mirroring the signal and application of spline interpolation that result in presence of sample at the zero crossing (bright dot).

interpolation of the signal in the time domain. The process of mirroring of the signal is illustrated in Figure 3.

If we have a very high quality signal without interference of additional waves the mirror image technique removes a portion of useful signal that can be used to determine the peak frequency (by cancelling incoherent noise). However, such arrivals are rare and if they occur the peak frequency of the full signal and mirrored signal are very consistent. To test how reduction of the signal influences obtained peak frequencies we apply mirror image technique to a synthetic dataset and benchmark it with results provided with FFT over full signal.

\section{TEST ON SYNTHETIC DATASET}

We construct synthetic waveforms corresponding to arrivals that resemble the Type 1 high quality - waveforms. The time function of the synthetic signal is using function of Fuchs and Mueller (1971) - symmetric signal with two extremes (peak and trough). These synthetic signals are superimposed with real noise from surface and downhole datasets. We use four different noise recordings; two from the downhole microseimic monitoring of the hydraulic stimulation of carbonate tight formation and shale, and two datasets acquired by surface arrays in West Bohemia (natural seismicity), Czech Republic (Fischer et al., 2014) and Agri Valley (possibly induced seismicity), South Italy (Stabile et al., 2014). The synthetic dataset simulating 
a)

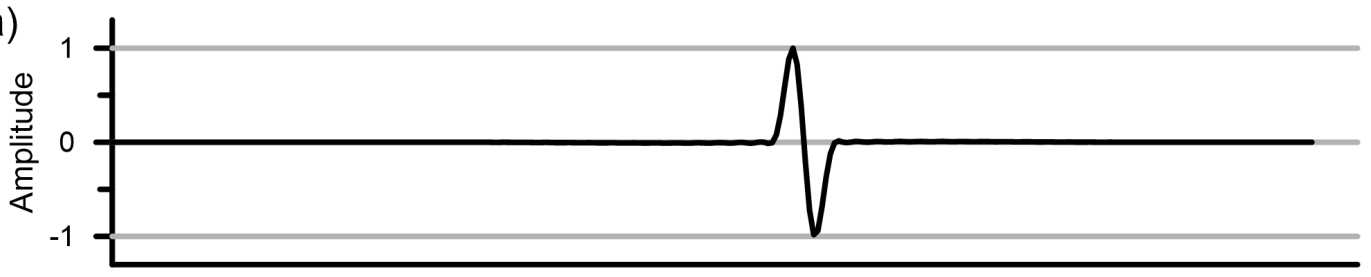

b)

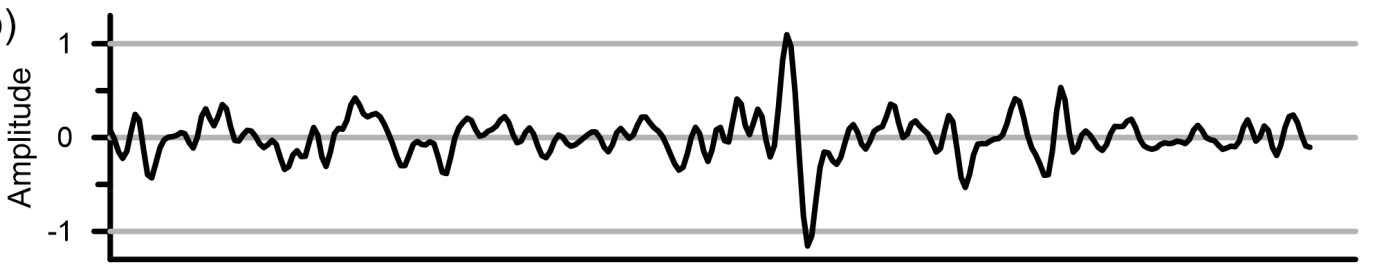

c)

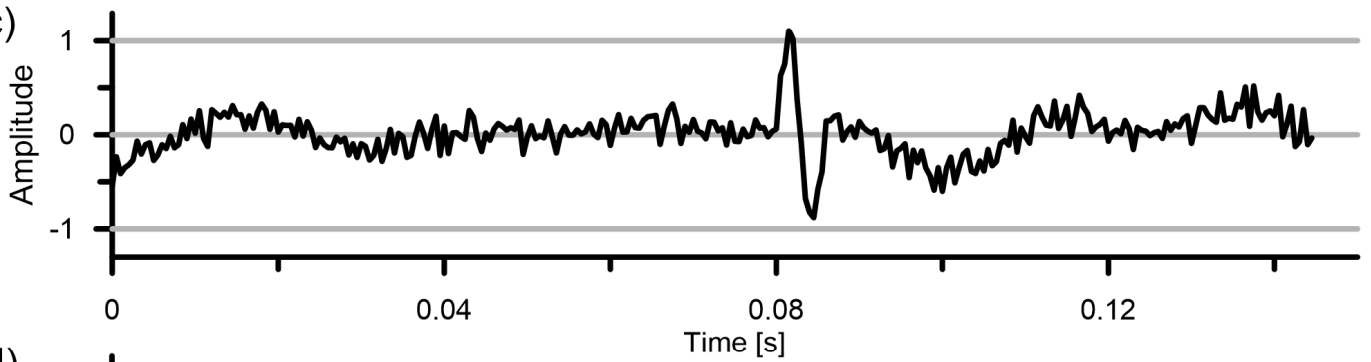

d)

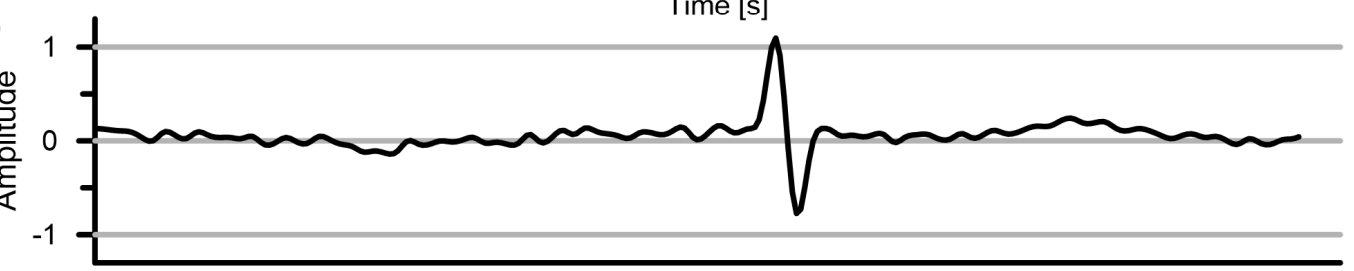

e)

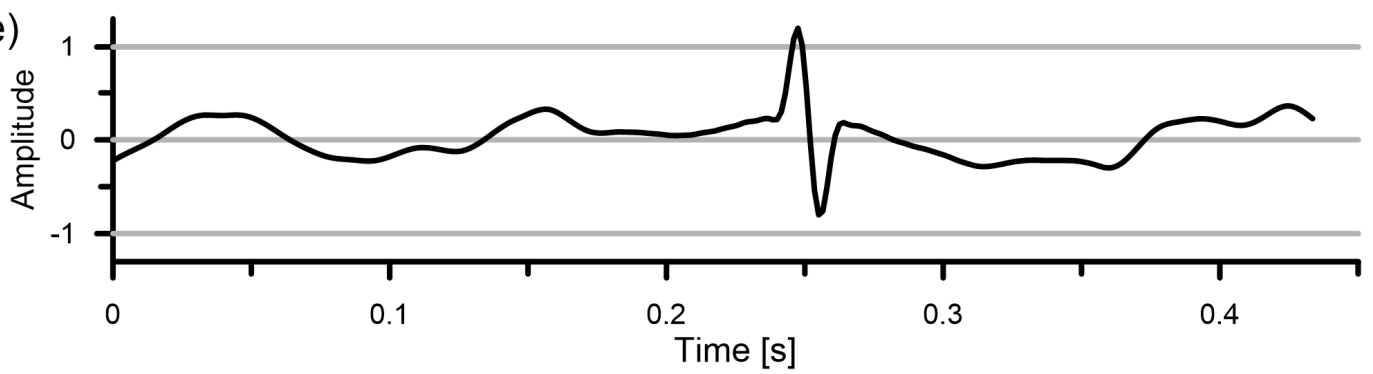

Fig. 4 Example of noise free and noise contaminated synthetic signal. a) Synthetic signal with $f_{\text {peak }}=135 \mathrm{~Hz}$ noise free, $b$ ) synthetic signal with $\mathrm{f}_{\text {peak }}=135 \mathrm{~Hz}$ and superimposed noise from downhole monitoring of carbonatite reservoir - noise level $30 \%$, c) synthetic signal with $\mathrm{f}_{\text {peak }}=135 \mathrm{~Hz}$ and superimposed noise from downhole monitoring of shale reservoir - noise level $30 \%$, d) synthetic signal with $\mathrm{f}_{\text {peak }}=45 \mathrm{~Hz}$ and superimposed noise from surface measurements from West Bohemia - noise level $30 \%$, e) synthetic signal with $\mathrm{f}_{\text {peak }}=45 \mathrm{~Hz}$ and superimposed noise from surface measurements from Agri Valley - noise level $30 \%$.

downhole signal used the peak frequency of $135 \mathrm{~Hz}$ to reflect real peak frequencies observed in the data. The synthetic dataset simulating signal registered at the surface used peak frequency of $45 \mathrm{~Hz}$ - the value that we can observe by monitoring with the use of surface networks. We simulate arrivals with different signal to noise ratio using level of noise equal $10 \%, 20 \%$ and $30 \%$. The noise level is the value of 90 th percentile of the absolute amplitude of noise samples relative to the peak amplitude of the signal samples. For example $30 \%$ of noise level means that 90th percentile of the noise samples is $30 \%$ of the peak amplitude of the signal. We created 100 realizations of the noise added to the signal (different intervals from long recoding of noise added to the signal). Noise free synthetic signals, as well as examples of signals with $30 \%$ noise level are shown in Figure 4.

Time intervals that were used to perform FFT over both mirrored and unaltered signal were selected manually to maintain consistency of picking. Each manually selected interval that indicate beginning and end of the arrival is extended by adding 3 unaltered and 5 samples with cosine taper applied before and after (in case of calculation of FFT over the whole signal) selected portion of the signal. It is standard procedure which purpose is to avoid spurious peaks in 
a)
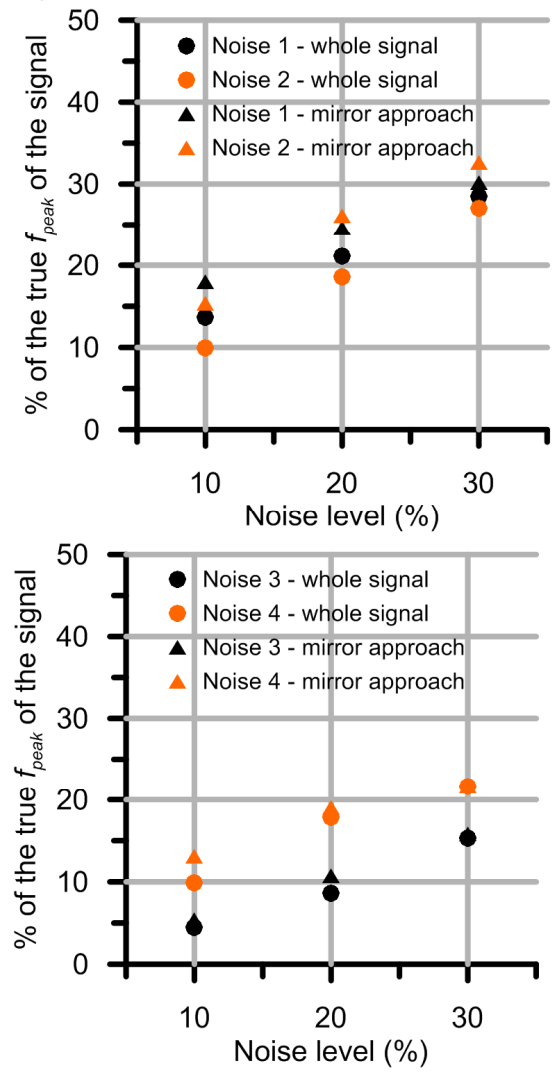

b)
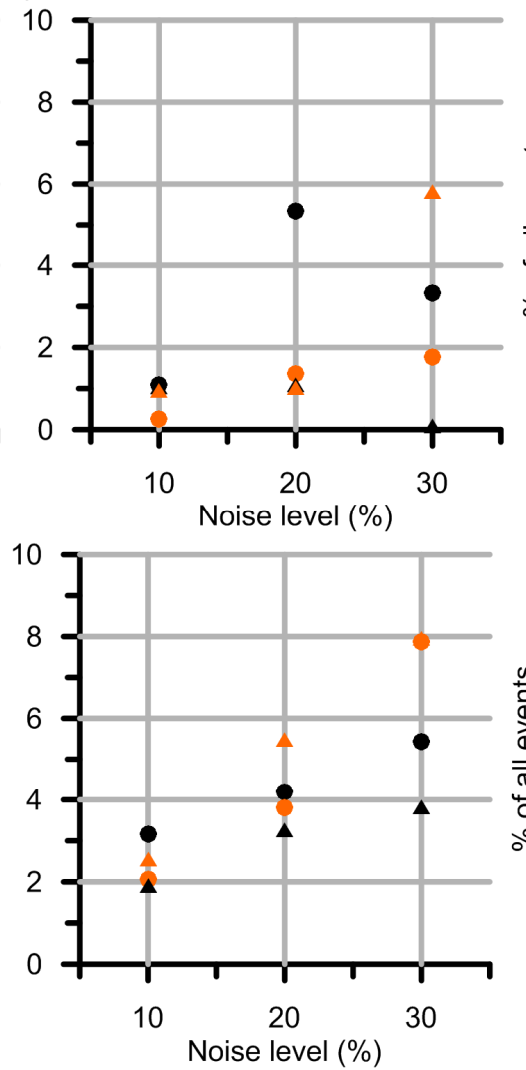

c)
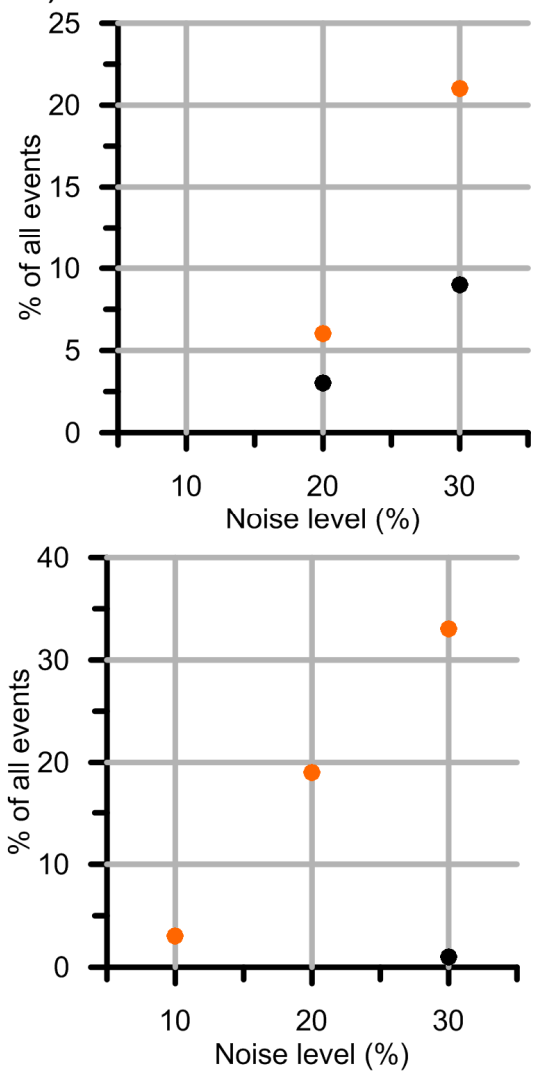

Fig. 5 Tests of determination of the peak frequency on synthetic datasets with different types of noise: noise $1-$ recordings from downhole monitoring of carbonatite reservoir, noise 2 - recordings from downhole monitoring of shale reservoir, noise 3 - surface recordings from the West Bohemia, noise 4 - surface recordings from Agri Valley; a) standard deviations of measured peak frequencies as a percentage of the $\mathrm{f}_{\text {peak }}$ of the noise free signal; b) absolute value of the difference between the median value of the measured peak frequencies in the each group and the true $f_{\text {peak }}$ of the synthetic signal; c) percentage of the signals that for FFT of the whole signal have resulted in $\mathrm{f}_{\text {peak }}=0$

the spectra caused by cutting the signal. The standard deviation as the percentage of the true peak frequency of the synthetic signal, difference between the median measured $\mathrm{F}_{\text {peak }}$ and the true peak frequency of the signal are shown in Figure 5. In the figure we also show how many measurements in case of use of whole signal (without mirroring), resulted in $\mathrm{F}_{\text {peak }}=0$. Those measurements were not included in calculation of median and standard deviation as they are clearly wrong and would significantly bias the statistics while no interpreter would take them into account.

The maximum absolute value of the error which indicates difference between median of measured peak frequencies and their real values is equal $8 \%$, while for sets with $10 \%$ and $20 \%$ noise level it is generally below $5 \%$. The standard deviations are higher when we use mirrored signal which is a result of the reduction of the useful signal used in measurements. For low noise levels the standard deviations of the measured peak frequencies for the mirror image methodology are approximately $50 \%$ higher. It means that to obtain stable value of effective $\mathrm{Q}$ we need higher number of measurements for the mirror technique. In case of higher noise the difference between both techniques is smaller. Moreover, we should note that the FFT over the whole signal results in some cases in $f_{\text {peak }}=0$, that further reduce statistical advantage of the use of the whole signal.

\section{REAL DATA EXAMPLE - WEST BOHEMIA DATASET}

We further test the performance of proposed methodology using real dataset from the swarm of natural earthquakes in the West Bohemia, Czech Republic. West Bohemia is seismically active region with intra-continental seismicity which has a form of seismic swarms with events of maximum magnitude $\mathrm{M}_{\mathrm{L}}$ usually below 4.0 (Fischer et al., 2014). The origin of the seismic swarm is probably related to upper mantle/lower crust fluid activity (Bräuer et al., 2009; Fischer et al., 2014). The area was already the object of the study concerning seismic attenuation by Wcisło et al. (2018) who used 2008 swarm data and utilized the manually determined half period technique of the peak frequency method in the determination of $Q_{P}$ 

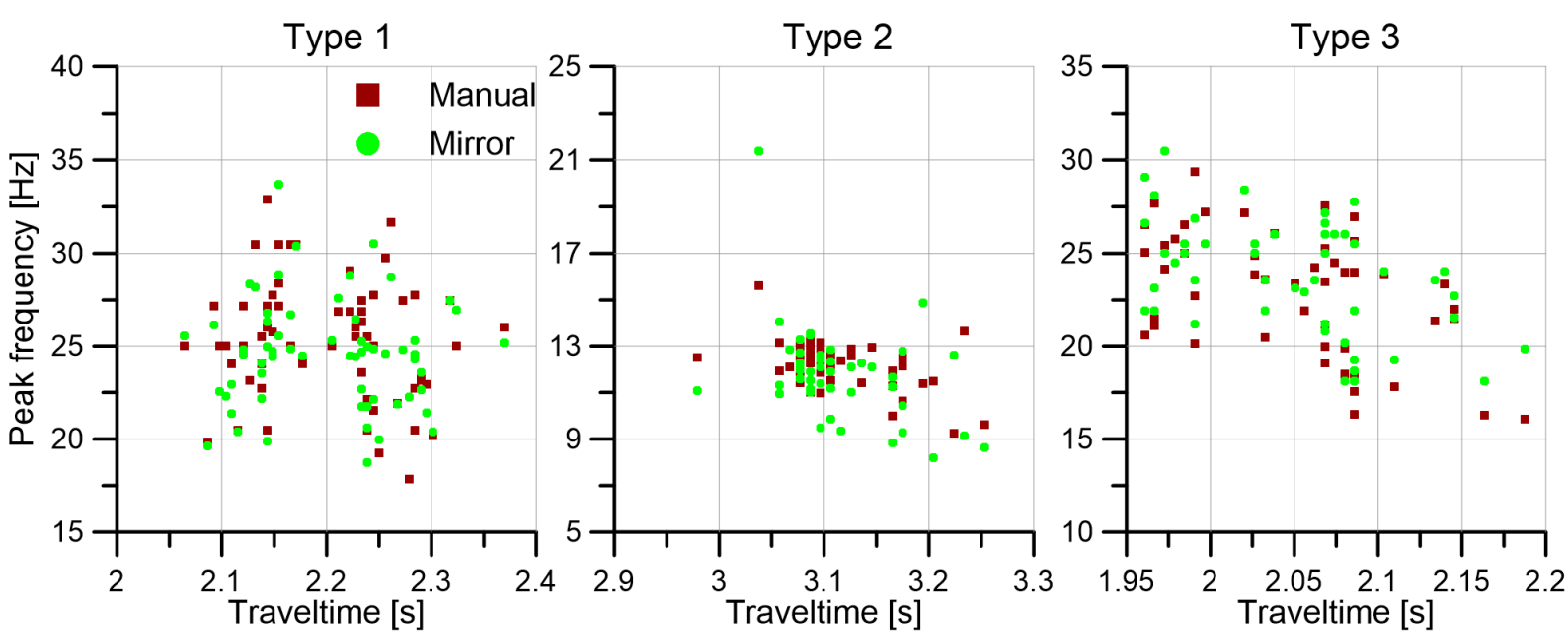

Fig. 6 Comparison of the measured peak frequencies using manual picking of first half period of the signal (squares) and the mirror technique (dots).

Table 1 Summary of the peak frequency measurements and inverted attenuation factors calculated for stations dominated by to the three different types of waveforms as illustrated in Figure 1 (Type 1: 60 arrivals, Type 2: 46 arrivals, Type 3: 49 arrivals).

\begin{tabular}{lcccccccc}
\hline Station & \multicolumn{2}{c}{$\begin{array}{c}\text { Median difference in } \mathrm{f}_{\text {peak }}(\%) \\
\text { compared to manual measurment }\end{array}$} & \multicolumn{3}{c}{ Median Q } & & \multicolumn{3}{c}{ Q standard deviation } \\
\cline { 2 - 9 } & $\begin{array}{c}\text { Mirror } \\
\text { technique }\end{array}$ & $\begin{array}{c}\text { Whole } \\
\text { signal }\end{array}$ & Manual & $\begin{array}{c}\text { Mirror } \\
\text { technique }\end{array}$ & $\begin{array}{c}\text { Whole } \\
\text { signal }\end{array}$ & Manual & $\begin{array}{c}\text { Mirror } \\
\text { technique }\end{array}$ & $\begin{array}{c}\text { Whole } \\
\text { signal }\end{array}$ \\
\hline Station 1 & 4.4 & 5.8 & 175 & 170 & 170 & 22 & 21 & 19 \\
Station 2 & 7.4 & 20.2 & 119 & 116 & 141 & 10 & 19 & 23 \\
Station 3 & 4.4 & 39.0 & 154 & 151 & 95 & 18 & 20 & 4 \\
\hline
\end{tabular}

and $\mathrm{Q}_{\mathrm{s}}$ in the region ( $\mathrm{Q}$ factors determined by Wcisło et al. (2018) are consistent with results of Mousavi et al. (2017)). We will use these manually picked peak frequencies as a benchmark for the automated procedure developed in this study.

For comparison we selected 3 stations (surface broadband seismometers) which during 2008 swarm (Horálek and Fischer, 2008) provided recordings with different level of interference of direct arrivals. Station 1 has direct arrivals of majority events without any interfering waves - the Type 1 waveform ( $\mathrm{P}$ waves). Station 2 has direct arrivals consistently similar to the Type 2 waveforms ( $\mathrm{S}$ waves) - small interference in the second part of the signal. Station 3 has direct arrivals corresponding to double arrival consistent with the Type 3 waveform ( $\mathrm{P}$ waves). Figure 6 shows values of measured peak frequencies for selected events using manual measurement of halfperiod in time domain and the Fourier transform of the mirror image of the signal. The measured peak frequencies are consistent for both types of measurement. The comparison of median obtained values of $\mathrm{Q}$ and their standard deviations for both techniques and FFT of the whole direct signal is shown in Table 1. The differences between medians of the peak frequencies for the half period and mirror technique are statistically insignificant for all stations, while the Fourier spectra of raw data provides reliable results only for the Type 1 recordings that corresponds to high quality waveforms without interference of later arrivals. Therefore, we may conclude that mirror technique provides a consistent measurement with manual processing. Note that mirror technique and manual picking of half-periods in time domain result in comparable standard deviations of the measured Q (elimination of two obvious outliers for the Station 2 waveforms in case of mirror technique measurements results in similar standard deviations for this station).

\section{MATCHED FILTER SELECTION OF EVENTS SUITABLE FOR AUTOMATED PEAK FREQUENCY DETERMINATION}

The use of the mirror technique to determine the peak frequency allows the automation of the peak frequency measurement. However, such automatic determination cannot be applied to all detected events as some events may have arrivals corrupted by noise or interfering waves. These complex waveforms cannot be used to reliably determine the peak frequency (and attenuation) as the frequency content of the direct arrival may result from complex wave propagation and interference (examples of such waveforms are shown in Figure 7). To overcome this issue we can select events by visual inspection of 
Table 2 Summary of inverted Q for manual and automatic procedure. The manual determination of quality factor is based on half-period technique and automated processing is based on correlation selection and mirror image techniaue.

\begin{tabular}{lcccc}
\hline \multirow{2}{*}{ Station } & \multicolumn{2}{c}{ Median Q } & \multicolumn{2}{c}{ Standard Deviation } \\
\cline { 2 - 5 } & Manual processing & Automatic processing & Manual processing & Automatic processing \\
\hline Station 1 & 175 & 174 & 22 & 20 \\
Station 2 & 119 & 116 & 10 & 10 \\
Station 3 & 154 & 148 & 18 & 18 \\
\hline
\end{tabular}

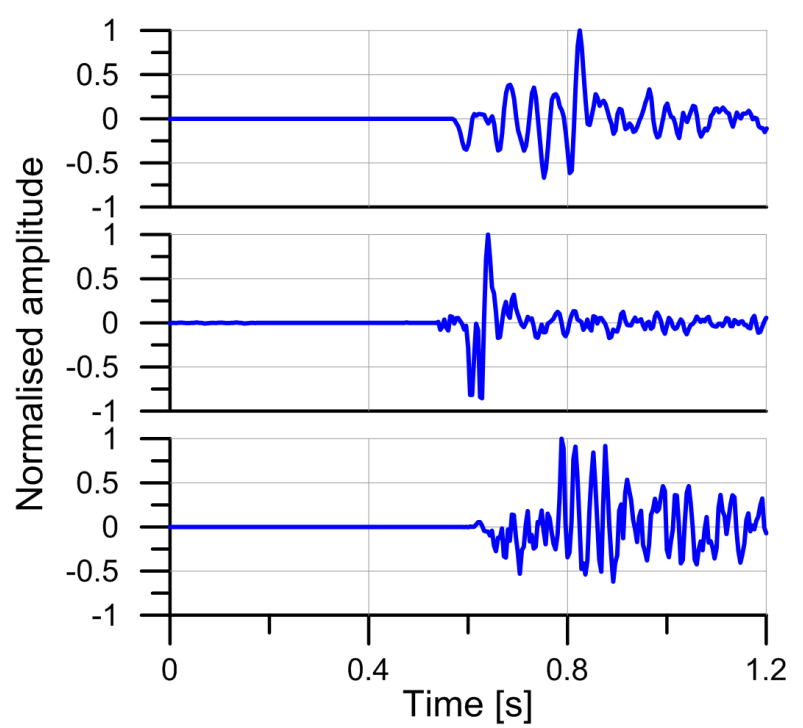

Fig. 7 Examples of high signal-to-noise ratio waveforms not suitable for determination of the peak frequency because they do not have distinguishable single direct arrival.

waveforms of detected events, which is the most reliable but also time consuming method. Alternatively we propose the use of the matched filter selection (Eisner et al., 2008; Caffagni et al., 2016). This selection uses template waveforms (so called Master events) to detect similar seismic events (e.g., Forghani-Arani et al., 2013) and is used in automatic picking by the industry (Schaff and Richards, 2014). If we select a template event with a clear simple arrival which is suitable for peak frequency mirror image determination, the matched filter will identify events with similar single arrival waveforms. It generally eliminates waveforms with complex arrivals as scattered arrivals after the first one will not correlate with the simple template waveform. As result cross correlation analysis may help in selection of waveforms suitable for determination of the peak frequency. The selection process is mostly reduced to the choice of the template event(s).

Another aspect of automatization is determination of the time interval used to calculate FFT of the mirrored signal. In principle it may be easily done by selecting significantly longer interval than the interval of the peak period of the master. In this way we should not cut the useful signal of selected events. Nevertheless, if the SNR is not high, it may significantly increase the scatter in the obtained frequencies. To limit the duration of the signal and therefore, influence of the noise we can follow 2 steps procedure:

1. First we can select long interval for calculations and proceed with determination of peak frequencies.

2. Refinement can be done with the new time intervals that correspond to the length of direct arrival calculated from values of peak frequency in the first step that is extended by a constant factor, e.g. factor of 1.5 or 2.0.

That ensures that each individual event would have customized length of interval used for calculations.

We test the determination of the peak frequency by the mirror technique on events selected by the matched filter. For each station used in the previous section we selected high SNR event with waveforms without guided and scattered waves. Specifically, in case of the Station 3 characterised by the most complex arrivals, we carefully selected an event with waveform from which the first half period of the direct arrival could be used for the peak frequency determination. The template interval was starting two peak periods before and two peak periods after the arrival. That prevents random oscillation to be picked by the filter. We used these templates to detect and cut events in 3 hours of continuous data. 75 detections with the highest value of correlation were selected for each station. They all included direct wave arrivals as multiple events were recorded during the investigated 3 hours.

We used these detections for the determination of the peak frequency by the mirror technique. In addition, for each station these 75 waveforms were visually inspected to ensure that waveforms were suitable for peak frequency method. Two waveforms were excluded from the analysis for the Station 1 and seven waveforms were excluded for the Station 3 as the result of visual inspection as they had the first part of the signal distorted. Note, that the visual inspection of only already matched events is much faster than selection from all events. Application of mirror technique was conducted according to the rules described in the previous parts of the study. Table 2 
Table 3 Results of analysis of the influence of master-event selection on the median $f_{\text {peak }}$ in the picked by crosscorrelation of waveforms.

\begin{tabular}{|c|c|c|c|c|c|c|c|c|}
\hline \multirow{2}{*}{$\begin{array}{l}\text { Station } \\
\text { Master event } \mathrm{f}_{\text {peak }}\end{array}$} & \multicolumn{4}{|c|}{ Station 1} & \multicolumn{4}{|c|}{ Station 3} \\
\hline & 21.1 & 25.2 & 30.5 & $\begin{array}{l}\text { Manual selection } \\
\text { (no master event) }\end{array}$ & 19.8 & 23.1 & 29.8 & $\begin{array}{l}\text { Manual selection } \\
\text { (no master event) }\end{array}$ \\
\hline $\mathrm{Nr}$ of measurments & 74 & 75 & 74 & 60 & 73 & 75 & 60 & 49 \\
\hline $\begin{array}{l}\text { Median measured } \\
f_{\text {peak }}\end{array}$ & 25.0 & 25.9 & 27.5 & 25.3 & 22.1 & 23.7 & 27.5 & 23.6 \\
\hline Standard deviation & 3.0 & 3.2 & 3.0 & 3.3 & 3.4 & 4.1 & 4.0 & 3.1 \\
\hline Max measured $f_{\text {peak }}$ & 30.5 & 32.8 & 35.9 & 32.9 & 32.0 & 32.8 & 35.9 & 30.5 \\
\hline Min measured $\mathrm{f}_{\text {peak }}$ & 19.1 & 19.8 & 21.4 & 17.9 & 18.3 & 16.8 & 19.1 & 18.1 \\
\hline
\end{tabular}

shows the results of this test in comparison to manually selected and picked events from Table 1 . The median $\mathrm{Q}$ values are consistent within the uncertainty of the measurement; additionally the uncertainties of both methods of measurements are comparable.

We note in passing that the matched filter detection may bias selection of events by filtering those with similar frequency content as crosscorrelation can be understood as filtering (in frequency domain). However, matched filters usually select repeated events from similar source location and such events produce waveforms resulting from similar attenuation effect as the locations are similar. Thus, if we consider microseismic events, the peak frequencies of those direct arrivals are similar. Nevertheless, we shall show on a real dataset that the use of matched filter does not significantly restrict selection of events and we still obtain events with significantly different values of $f_{\text {peak }}$. To do it we selected several waveforms with single arrivals which were used as master events for the Stations 1 and 3. These stations are characterised by the higher standard deviation of the attenuation measurement which indicates higher variability of the signal. Each selected template event had a different peak frequency: one higher, one lower and one similar to the median $\mathrm{f}_{\text {peak }}$ of manually selected waveforms. For each Master event, we took 75 matched filter detections with the highest correlation coefficient. We have excluded obviously distorted events with interference in the first half period of the signal (especially for Station 3). Table 3 summarizes results of this test which provides following conclusions:

- peak frequency of master event affects the median peak frequencies of the selected set of events,

- the median peak frequency of the events detected with given Master event with higher or lower value of the $f_{\text {peak }}$ than the average is closer to average than the peak frequency of the Master event itself,

- for investigated dataset the use of the Master event does not result in reduced standard deviation - we still select events with variety of peak frequencies with significant difference between minimum and maximum measured peak frequency.

Note that difference in median peak frequency of selected events does not linearly translate into difference in calculated attenuation coefficient $\mathrm{Q}$ as the calculation includes both peak frequency as well as the traveltime (Eq. 2).

Based on the tests of the matched filter selection we propose the following steps in automated determination of attenuation from a dataset of microseismic events:

1. Select high-quality Master event from a catalogue.

2. Use correlation detection to select group of highquality events that are likely suitable for determination of the peak frequency using mirror technique.

3. Inspect visually detected events and select events that are suitable (cut unsuitable events).

4. Determine peak frequencies of the detected events.

5. Repeat steps 1-4 for various masters.

Proposed workflow with the use of different masters allows selection of a broad group of events with range of measured peak frequencies which represent given dataset. In case of noisy datasets, it allows selection of suitable waveforms, without timeconsuming manual preselection. This procedure limits influence of the interpreter on the results of measurements as well.

\section{DOWNHOLE MONITORING DATASET}

We test the proposed workflow using a dataset acquired during hydraulic fracturing of Permian basin Wichita Albany in Texas, United States that took place in 2015. Hydraulic fracturing was made in dolomite rocks which were fractured in 21 stages. Stages 9-14 were used for this study as they were the closest to the monitoring borehole. Monitoring array consisted of 24 downhole geophones (sampling frequency $4000 \mathrm{~Hz}$ ) which were located in a vertical borehole. We used events from stage 12 as it had the 


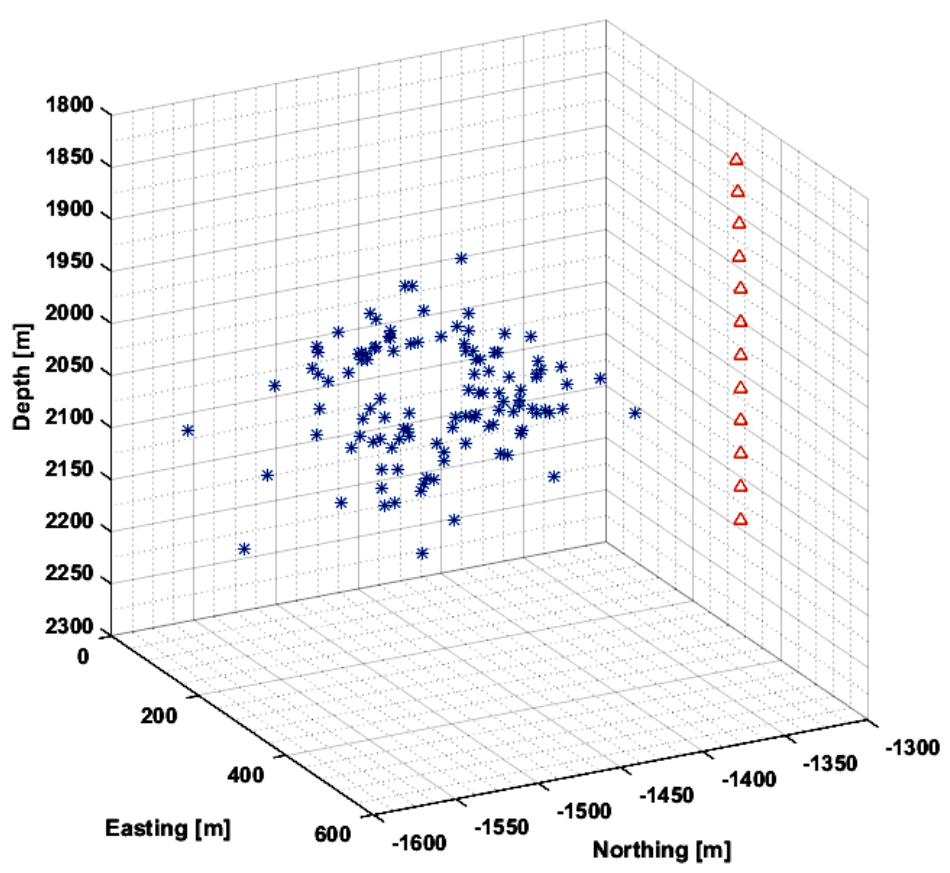

Fig. 83 D view of geophones and stage 12 events location (there is a pair of 2 geophones at each given depth). Events are represented by asterisks, geophone locations are represented by triangles.

Table 4 Measured $\mathrm{Q}_{\mathrm{S}}$ for the hydraulic fracturing dataset.

\begin{tabular}{lcccc}
\hline Geophone & \multicolumn{2}{c}{ Median measured Q } & \multicolumn{2}{c}{ Standard deviation } \\
\cline { 2 - 5 } & Manual & Automatic & Manual & Automatic \\
\hline No. 1 & 176 & 187 & 42 & 41 \\
No. 4 & 153 & 165 & 37 & 45 \\
No. 6 & 172 & 188 & 41 & 46 \\
\hline
\end{tabular}

highest number of detected events. Event locations from this stage are shown in Figure 8. Average distance between the closest receiver and events is approximately 300 meters. We measure effective $\mathrm{S}$-waves attenuation only because $\mathrm{P}$-wave signal to noise ratio is too low for reliable measurement.

After brief visual inspection we selected 3 receivers - Receivers 1, 4 and 6 , whose recordings contained a number of waveforms. We need to note that majority of the registered events were not suitable for the determination of peak frequencies which would make manual selection of waveforms particularly time consuming. For each receiver we selected a clear waveform (not necessarily of the same event) which was used as the Master in matched filter detection of approximately 2 hours of continuous data. This way for each receiver we selected 50 matched filter detections with highest value of cross-correlation. We inspected them to exclude waveforms which have first half period distorted or do not correspond to the direct arrivals. For Receiver 1 we removed 7 events, for Receiver 4 - we removed 6 events and for Receiver 6 - we removed 8 events. Selected Master events and examples of waveforms picked by the above selection process are shown in Figure 9. The peak frequencies calculated using selected waveforms were used for determination of attenuation factor. Summary of the calculated $\mathrm{Q}_{\mathrm{S}}$ using automatic mirror technique and manual picking of first half period is presented in Table 4.

Similarly to the case study of the West Bohemia dataset the calculated attenuation is very similar for manual and automated technique. Median values do not differ by more than $10 \%$. Lower attenuation factor (hence lower measured peak frequencies) of Swaves for all 3 geophones calculated using manual technique may result from interpreter's picking patterns of selection of the onset. Standard deviations of measured attenuation quality factors are higher than in the case of West Bohemia, nevertheless they are still relatively low for attenuation inversion (Tonn, 1989). The scatter may also result from attenuation heterogeneity of the layered formation or may be caused by hydraulic fracturing or attenuation anisotropy (e.g., Červený and Pšenčík, 2008). Measured $Q$ is high in comparison to majority of reservoirs (Delle Piane et al., 2014; Shekar and Tsvankin, 2012), nevertheless obtained values are consistent with results of Adam et al. (2009) who analysed attenuation of dolomites in slightly higher 

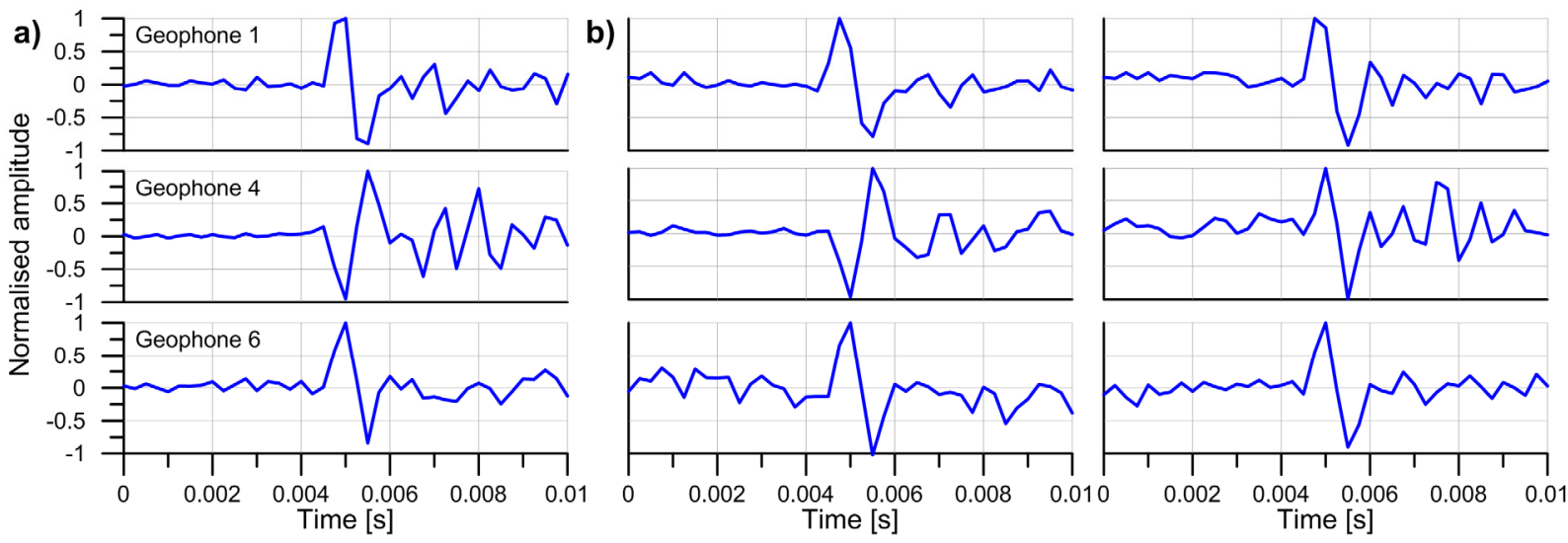

Fig. 9 a) Raw waveforms of Master events (1 for each selected geophone) used for cross-correlation analysis; b) examples of two detected by cross-correlation waveforms for each selected geophone.

bandwith (on average $500-800 \mathrm{~Hz}$ in our case). Note that the frequency of the master-event did not resulted in biased selection as master events have calculated peak frequency 770, 780 and $680 \mathrm{~Hz}$ and median peak frequency of the waveforms picked by crosscorrelation are 585, 670 and $675 \mathrm{~Hz}$ with standard deviation of 135, 165 and $120 \mathrm{~Hz}$ respectively.

\section{CONCLUSIONS}

We have developed the mirror technique to calculate the peak frequency and attenuation from microseismic events. We show that this technique is consistent with the manual processing using halfperiod technique. Use of peak frequency values obtained from the Fourier Spectra analysis of mirrored signal excludes subjectivity of the manual picking of the first half-period. Additionally we show that the matched filter detections may be used to automatically select the waveforms suitable for automated processing. The combination of these two methods provides technique of quick semi-automatic Q determination.

\section{ACKNOWLEDGMENTS}

This work was carried out thanks to the support of the long-term conceptual development research organization RVO: 67985891. It was also supported by Charles University grant SVV 260447 . We thank Institute of Rock Structure and Mechanics CAS and Institute of Geophysics CAS for sharing the data from WEBNET seismic network.

\section{REFERENCES}

Adam, L., Batzle, M., Lewallen, K. and van Wijk, K.: 2009, Seismic wave attenuation in carbonates. J. Geophys. Res., Solid Earth, 114, No. B6. DOI: $10.1029 / 2008 J B 005890$

Beckwith, J., Clark, R. and Hodgson, L.: 2017, Estimating frequency-dependent attenuation quality factor from prestack surface seismic data. Geophysics, 82, 1, 011022. DOI: 10.1190/geo2016-0169.1
Bräuer, K., Kämpf H. and Strauch, G.: 2009, Earthquake swarms in non-volcanic regions: What fluids have to say. Geophys Res. Lett., 36, No. 17, L17309. DOI: $10.1029 / 2009$ GL039615

Castiello, A., Ren, Y., Greenwood, S., Martin, T., Luo, Z. and Buchan, I.: 2015, Seismic imaging in gas obscured areas - Q anomaly detection and Q migration applied to broadband data. 77th EAGE Conference \& Exhibition, Extended Abstract, N107 11. DOI: 10.3997/2214-4609.201413252

Caffagni, E., Eaton, D., Jones, L. and van der Baan, M.: 2016, Detection and analysis of microseismic events using a Matched Filtering Algorithm (MFA). Geophys. J. Int., 206, No. 1, 644-658. DOI: $10.1093 / \mathrm{gji} / \mathrm{ggw} 168$

Červený, V.: 2001, Seismic ray theory. Cambridge University Press, Cambridge, United Kingdom.

Červený, V. and Pšenčík, I.: 2008, Quality factor Q in dissipative anisotropic media. Geophysics, 73, No. 4, 63-75. DOI: 10.1190/1.2937173

Delle Piane, C., Sarout, J., Madonna, C., Seanger, E., Dewhurst, D. and Raven, M.: 2014, Frequencydependent seismic attenuation in shales: experimental results and theoretical analysis. Geophys. J. Int., 198, No. $1,504-515$. DOI: $10.1093 /$ gji $/$ ggu148

Eisner, L., Abott, D., Barker, W., Lakings, J. and Thornton, M.: 2008, Noise suppression for detection and location of microseismic events using a matched filter. SEG Technical Program Expanded Abstracts 2008, 14311435. DOI: $10.1190 / 1.3059184$

Eisner, L., Gei, D., Hallo, M., Opršal, I. and Ali, M.Y.: 2013, The peak frequency of direct waves for microseismic events. Geophysics, 78, No. 6, 45-49. DOI: 10.1190/geo2013-0197.1

Fischer, T., Horálek, J., Hrubcová, P., Vavryčuk, P., Bräuer, K. and Kämpf, H.: 2014, Intra-continental earthquake swarms in West-Bohemia and Vogtland: A review. Tectonophysics, 611, 1-27. DOI: $10.1016 /$ j.tecto.2013.11.001

Forghani-Arani, F., Behura, J., Haines, S. and Batzle, M.: 2013, An automated cross-correlation based event detection technique and its application to a surface passive data set. Geophys. Prospect., 61, No. 4, 778787. DOI: $10.1111 / 1365-2478.12033$

Fuchs, K. and Müller, G.: 1971, Computation of synthetic seismograms with the reflectivity method and 
comparison with observations. Geophys. J. Int., 23, No. 4, 417-433.

DOI: 10.1111/j.1365-246X.1971.tb01834.x

Huang, G., Chen, X., Luo, C., Li, J. and Dai, H.: 2018, AVA inversion based on the wave equation with attenuation compensation. SEG Technical Program Expanded Abstracts, 620624. DOI: 10.1190/segam2018-2994004.1

Guo, P. and McMechan, G.: 2017, Sensitivity of 3D 3C synthetic seismograms to anisotropic attenuation and velocity in reservoir models. Geophysics, 82, No. 2, T79-T95.

DOI: $10.1190 /$ geo2016-0321.1

Hallo, M.: 2012, Microseismic surface monitoring network design - Sensitivity and accuracy. 74th EAGE Conference \& Exhibition Extended Abstract, P021.

DOI: $10.3997 / 2214-4609.20148336$

Horálek, J. and Fischer, T.: 2008, Role of crustal fluids in triggering the West Bohemia/Vogtland earthquake swarms: just what we know (a review). Stud. Geoph. Geod., 52, No. 4, 455478. DOI: $10.1007 / \mathrm{s} 11200-008-0032-0$

Jechumtálova, Z. and Eisner, L.: 2008, Seismic source mechanism inversion from linear array of receivers reveal non-double-couple seismic events induced by hydraulic fracturing in sedimentary formation. Tectonophysics, 460, 124-133. DOI: 10.1016/j.tecto.2008.07.011

Kjartansson, E.: 1979, Constant Q-wave propagation and attenuation. J. Geophys. Res. - Solid Earth, 84, No. B9, 4737-4748. DOI: $10.1029 / J B 084 i B 09 p 04737$

Matheney, M.P. and Nowack, R.L.: 1995, Seismic attenuation values obtained from instantaneousfrequency matching and spectral ratios. Geophys. J. Int., 123, No. 1, 1-15. DOI: 10.1111/j.1365-246X.1995.tb06658.x

Mousavi, S., Haberland, C., Bauer, P., Hejrani, B. and Korn, M.: 2017, Attenuation tomography in West Bohemia/Vogtland. Tectonophysics, 695, 64-75. DOI: 10.1016/j.tecto.2016.12.010

Pham, N., Carcione, J., Helle, H. and Urisn, U.: 2002, Wave velocities and attenuation of shaley sandstones as function of pore pressure and partial saturation. Geophys. Prospect., 50, No. $6,615-627$.

DOI: 10.1046/j.1365-2478.2002.00343.x

Quan, Y. and Harris, J.M.: 1997, Seismic attenuation tomography using the frequency shift method. Geophysics, 62, No. 3, 895-905.

DOI: $10.1190 / 1.1444197$
Schaff, D. and Richards, P.G.: 2014, Improvements in magnitude precision, using the statistics of relative amplitudes measured by cross correlation. Geophys. J. Int., 197, No. 1, 335-350. DOI: 10.1093/gji/ggt433

Shekar, B. and Tsvankin, I.: 2012, Anisotropic attenuation analysis of crosshole data generated during hydraulic fracturing. Lead. Edge, 31, No. 5, 588-593.

DOI: $10.1190 /$ tle 31050588.1

Stabile, T.A., Giocoli, A., Perrone, A., Piscitelli, S. and Lapenna, V.: 2014 Fluid injection induced seismicity reveals a NE dipping fault in the southeastern sector of the High Agri Valley (southern Italy). Geophys. Res. Lett., 41, No. 16, 5847-5854. DOI: $10.1002 / 2014$ GL060948

Tonn, R.: 1989, Comparison of seven methods for the computation of Q. Phys. Earth Planet.y Inter., 55, No. 3-4, 259-268, DOI: 10.1016/0031-9201(89)90074-5

Tselentis, G., Paraskevopoulos, P. and Martakis, N.: 2010, Intrinsic Qp seismic attenuation from the rise time of microearthquakes: a local scale application at RioAntirrio, Western Greece, Geophys. Prospect., 58, No. 5, 845-859. DOI: 10.1111/j.1365-2478.2010.00885.x

Wcisło, M. and Eisner, L.: 2016, Attenuation from microseismic datasets by the peak frequency method benchmarked with the spectral ratio method. Stud. Geoph. Geod., 60, No. 3, 547-564. DOI: $10.1007 / \mathrm{s} 11200-015-0577-7$

Wcisło, M., Eisner, L., Malek, J., Fischer, T., Vlcek, P. and Kletetschka, G.: 2018, Attenuation in West-Bohemia: evidence of high attenuation in the Nový Kostel focal zone and temporal changes consistent with $\mathrm{CO} 2$ degassing. Bull. Seis. Soc. Am., 108, No. 1, 450-458. DOI: $10.1785 / 0120170168$

Wüstefeld, A., Lecomte, I., Oye, V., Kühn, D. and Kværna, T.: 2014, Quantitative network design optimization. 5th EAGE Passive Seismic Workshop. DOI: $10.3997 / 2214-4609.20142171$ 